\title{
Aspectos biopsicosociales en la valoración de la dependencia
}

\section{Bio-psychosocial aspects in care need assesment}

\section{G. GÓMEZ-JARABO \\ J. C. PEÑALVER GONZÁLEZ ${ }^{2}$}

\section{RESUMEN}

En el presente trabajo hemos partido de la concepción politica de la dependencia desde el Consejo de Europa, nos parece un acierto su definición psicosocial y su marco de atención sociosanitaria, es por su parte axiomático que a la dependencia se llega por la edad, la discapacidad o la combinación de ambas condiciones, de tal manera que independientemente del motivo lo que aparece es una población social que tiene dificultades en la realización de la actividades básicas e instrumentales de la vida diaria y que son tanto más dependientes cuanto mayor son los cambios de las estructuras sociales de convivencia y tanto más vulnerables cuanto menos desarrollados estén los servicios sociales y en particular el denominado espacio sociosanitario. Hemos hecho referencia a la prospectiva que la Encuesta sobre Deficiencias, Discapacidades y Estados de Salud de 1999 nos ofrece y consideramos que de no desarrollarse un eficaz Sistema Nacional de Dependencia el éxito biológico alcanzado en el pasado siglo va a ser sin duda seguido de un incuestionable fracaso social.

\section{PALABRAS CLAVE}

Dependencia, Discapacidad y edad. \footnotetext{
UAM.

Profesor titular de Psicobiología y Director del Máster en Valoración de Discapacidades e Incapacidad Laboral

${ }^{2}$ Profesor invitado del Máster en Valoración de Discapacidades e Incapacidad Laboral.
} 
Aspectos biopsicosociales en la valoración de la dependencia

\section{ABSTRACT}

This paper adopts the Council of Europe political view of care need. This is defined in psychosocial terms and takes place within a socio-medical framework. Obviously, people may be in need of care for a number of reasons such as becoming old or with disability or a combination of both situations. Thus, regardless of these reasons, what appears is a population having difficulties to get by with basic daily activities. Therefore, the bigger the change in the social structures of the living-together, the higher the need of care. Likewise, the less developed the social services, particularly the so-called socio-medical space, the more vulnerable the people in need of care. With respect to the prospects of the1999 Survey on Deficiencies, Disabilities and Health Conditions, we think that if an efficient National Care Need System is not developed the biological success attained in the past century will be followed by a resounding social failure.

\section{KEY WORDS}

Care Need, Disability, Ageing. 


\section{INTRODUCCIÓN}

El Consejo de Europa, constituyó a mediados de la década de los noventa un grupo de expertos que trabajó durante dos años en la elaboración de un texto de consenso que pudiese ser asumido desde la mayoría de los países miembros de este organismo internacional. El resultado de ese trabajo fue la aprobación, en septiembre de 1998, por el Comité de Ministros del Consejo de Europa, de una recomendación relativa a la dependencia (Consejo de Europa, 1998).

En este documento el Consejo de Europa define la dependencia como "la necesidad de ayuda o asistencia necesaria para realizar las actividades de la vida cotidiana”, o, de manera más precisa, como "un estado en el que se encuentran las personas que por razones ligadas a la falta o la pérdida de autonomía física, psiquica o intelectual, tienen necesidad de asistencia y/o ayudas importantes a fin de realizar los actos corrientes de la vida diaria $y$, de modo particular, los referentes al cuidado personal".

Esta definición, que ha sido ampliamente aceptada, plantea la concurrencia de tres factores para que podamos hablar de una situación de dependencia: en primer lugar, la existencia de una limitación física, psiquica o intelectual que merma determinadas capacidades de la persona; en segundo lugar, la incapacidad de la persona para realizar por si mismo las actividades de la vida diaria; e incluso de las actividades, llamadas instrumentales de la vida diaria; en tercer lugar, la necesidad de asistencia 0 cuidados por parte de un tercero.

La dependencia puede entenderse, por tanto, como el resultado de un proceso que se inicia con la aparición de un défi- cit en la estructura o en el funcionamiento de alguna parte de nuestro cuerpo (aspecto biológico), lo cual comporta una limitación en la actividad.

Cuando esta limitación no puede compensarse mediante la adaptación personal o del entorno, provoca una restricción en la participación social del individuo que se concreta en la dependencia de la ayuda de otras personas para realizar las actividades de la vida cotidiana (aspecto social).

La evidencia empírica disponible muestra que existe una estrecha relación entre dependencia y edad, pues el porcentaje de individuos con limitaciones en su capacidad funcional aumenta conforme consideramos grupos de población de mayor edad. Ese aumento en las tasas de prevalencia por grupos de edad no se produce a un ritmo constante, sino que existe una edad (alrededor de los 80 años) en que dicho aumento se acelera notablemente. No es extraño, por ello, que la dependencia se vea en ocasiones, como un problema estrechamente vinculado al envejecimiento demográfico, y que, algunas veces, en una visión reduccionista, se tienda a considerar la dependencia como un fenómeno que afecta sólo a los mayores. En realidad, la dependencia recorre todas las edades de la población. No se puede circunscribir, por ello, el fenómeno de la dependencia al colectivo de las personas mayores, aun cuando sean éstas las que con más intensidad se ven afectadas. La dependencia puede aparecer en cualquier momento de nuestra vida.

Puede estar presente desde el nacimiento, desencadenarse a consecuencia de un accidente automovilístico o de una enfermedad aguda en el periodo pre natal 0 post natal o en la infancia, la juventud o la vida adulta o, más frecuen- 
temente, ir apareciendo a medida que las personas envejecen, como consecuencia de enfermedades crónicas (demencias, artritis, trastornos visuales, etc.)

La dependencia puede también no aparecer, y de hecho en muchos casos no aparece aunque la persona haya alcanzado una edad muy avanzada. Existen por tanto una serie de variables de tipo biológico, social y ambiental (además de los factores genéticos), que condicionan la aparición y el desarrollo de los desencadenantes de la dependencia, y en las que los individuos difieren entre sí. De lo anterior se deriva una conclusión: la dependencia puede detenerse 0 limitarse promoviendo hábitos de vida saludables, mejorando la eficacia de los sistemas de atención de la salud y asegurando el tratamiento precoz de las enfermedades crónicas.

En España sin embargo, el proceso acelerado de envejecimiento de la población está originando una situación problemática, tanto cuantitativa como cualitativamente, al coincidir este envejecimiento acelerado con cambios profundos en la estructura social de la familia y de la población cuidadora.

Obviamente, la necesidad de asistencia y/o cuidados derivada de las situaciones de dependencia ha existido siempre; lo que ha cambiado es su dimensión, su importancia social, ha dejado de verse como un problema exclusivamente individual o familiar y su naturaleza implica nuevos compromisos de protección y financiación, en las funciones del Estado del Bienestar.

Las personas dependientes requieren una intervención continuada, aunque no siempre permanente, basada en el apoyo y los cuidados. La atención de la dependencia se enmarca, por tanto, en el ámbito de los servicios sociales. Sin embargo, existe una fuerte relación con el ámbito biológico sanitario, pues:

- Un problema de salud que precise atención sanitaria puede ser el factor desencadenante 0 acelerador de los procesos de dependencia en las personas de edad avanzada.

- De forma ocasional, la persona dependiente puede sufrir un proceso agudo en su estado de salud que requiera una atención sanitaria puntual.

La creación y existencia de una red adecuada para dar cobertura a los casos de dependencia en su vertiente social descargaría el sistema sanitario de un gran volumen de costes, que son, por otra parte, desproporcionados en relación con la atención que las personas dependientes necesitan.

Existe una clarísima interrelación entre los aspectos biológicos del individuo y las situaciones de dependencia que puede llegar a padecer. En concreto, se tiene constancia de la eficacia de las intervenciones sanitarias en edades medianas de la vida para prevenir la aparición de la dependencia en las edades más avanzadas, y se ha demostrado, asimismo, que la mejora de los hábitos de vida de la población contribuye significativamente a mejorar la esperanza de vida sin discapacidad. Esto confiere a la intervención sanitaria un importante papel, al atrasar la aparición de la dependencia.

Por otra parte, en los casos en que la dependencia ya está establecida, el cuidado de la salud es esencial para lograr una adecuada adaptación de la persona a su nueva situación y mejorar su calidad de vida. De hecho, la más que frecuente coexistencia de enfermedades activas complica la situación, hasta el 
punto que los casos de personas mayores con necesidades asistenciales, son aquellas en las que la situación de dependencia funcional coincide con la pluripatologia y la inestabilidad clínica.

\section{CONCEPTO DE "ESPACIO SOCIOSANITARIO"}

Actualmente existe una asimetría entre los servicios sanitarios y los sociales y esto es precisamente, una de las dificultades más significativas para la protección de las personas con dependencia, pues mientras el cuidado de la salud constituye un servicio de carácter universal y gratuito como derecho legal para todos los ciudadanos, los servicios sociales públicos todavia se entienden con un carácter graciable y un desarrollo desigual.

Organizar y coordinar ambos tipos de servicios es esencial para que el país pueda proporcionar una respuesta integral y eficaz. La demanda de cuidados para personas dependientes se ha venido incrementando de forma notable en los últimos años y va a seguir aumentando a un fuerte ritmo durante las próximas décadas, como consecuencia de la combinación de factores de carácter demográfico, sanitario y social, entre los que podemos citar el envejecimiento de la población, las mayores tasas de supervivencia de las personas afectadas por alteraciones congénitas, enfermedades y accidentes graves, y la creciente incidencia de los accidentes laborales y de tráfico.

Ese crecimiento de las demandas coincide en el tiempo con la crisis de los sistemas de apoyo informal que tradicionalmente han venido dando respuesta a las necesidades de cuidados. Una crisis motivada, fundamentalmente, por los cambios en el modelo de familia y la cre- ciente incorporación de las mujeres al mundo laboral. El proceso de envejecimiento demográfico incrementa las necesidades de cuidados y atención En las últimas décadas la población mayor española ha experimentado un crecimiento muy considerable.

El número de personas mayores de 65 años se ha duplicado en los últimos treinta años del siglo XX, ha pasado de 3,3 millones de personas en 1970 (un 9,7 por ciento de la población total) a más de 6,6 millones en 2000 (16,6 por ciento de la población total).

Este fenómeno del envejecimiento va a continuar en los próximos años, en los que la población mayor seguirá incrementándose de manera notable, a la vez que disminuirá la proporción de personas en edades jóvenes, como muestran la evolución prevista de la estructura de la población española durante el período 1991-2026 (INE).

Durante los primeros años del siglo XXI, el envejecimiento de la población está teniendo unas características peculiares, condicionadas por la historia demográfica española. La razón es la caída de la natalidad que se produjo en España en los años que siguieron a 1936 Los miembros de esas generaciones relativamente poco numerosas nacidas en los años de la Guerra Civil han comenzado a incorporarse a la población mayor de sesenta y cinco años a partir del año 2001.

Como resultado, se está produciendo, en esta primera década del siglo, una apreciable disminución del ritmo de crecimiento de la población mayor en su conjunto y un leve descenso en el número de personas que cumplen cada año la edad de jubilación, que ha aliviado un poco la presión que ha venido ejerciendo el crecimiento continuado de la población mayor sobre los sistemas de pensiones. 
Aspectos biopsicosociales en la valoración de la dependencia

Grafico 1. Proyecciones de la estructura por grandes grupos de edad de la población española, 1991-2006

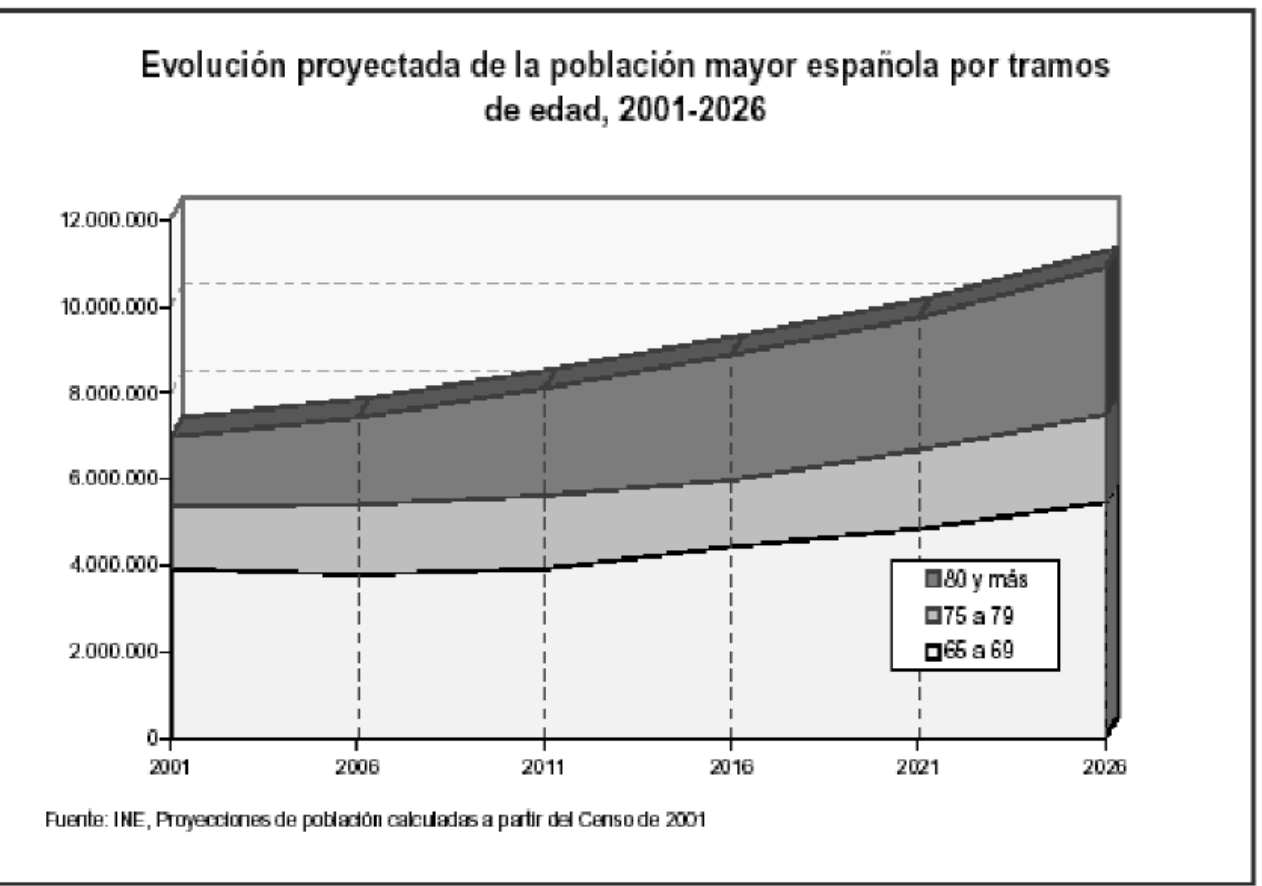

Desde esta perspectiva, estamos viviendo una especie de "tregua demográfica” para el sistema de Bienestar Social. Sin embargo, junto a esa ralentización del crecimiento de la población mayor en su conjunto, se está produciendo un incremento muy considerable del número de personas mayores de 80 años (el grupo de población en el que se concentran la mayor parte de las situaciones de dependencia), que aumentará en 850.000 personas en esta década; es decir, que gracias a la tregua demográfica, el éxito biológico alcanzado en el pasado siglo no es todavía causa de un fracaso social.

La pretendida "tregua" no es, pues, más que una ilusión, pues la presión sobre los recursos no va a desaparecer, sino que simplemente se desplazará desde las prestaciones económicas hacia las prestaciones de servicios, y va a exigir un importante esfuerzo inversor para poner al dia nuestra insuficiente red asistencial.

A partir de 2011 el crecimiento global de la población mayor volverá a intensificarse, debido a la incorporación de generaciones más numerosas. Ese crecimiento alcanzará una particular intensidad a partir del año 2020, en el que empezarán a incorporarse a la población mayor las primeras generaciones del "baby-boom", que en España comenzó a producirse a finales de los años cincuenta. (Atención a las personas en situación de dependencia en España Libro Blanco. IMSERSO).

Tradicionalmente, han sido las familias las que han asumido el cuidado de 
las personas dependientes, a través de lo que ha dado en llamarse "apoyo informal”. Para ser más exactos, habría que puntualizar que esa función ha recaído y recae, casi en exclusiva, en las mujeres del núcleo familiar (esto es, en las madres, cónyuges, hijas o hermanas de las personas dependientes), y, dentro de éstas, en las mujeres de mediana edad, sobre todo en el grupo formado por las que tienen entre 45 y 69 años.

El incremento cuantitativo de las situaciones de dependencia, motivado por el envejecimiento de la población y por el incremento de la morbilidad, coincide en el tiempo con cambios importantes en el modelo de familia y con la incorporación progresiva de la mujer al mercado de trabajo, fenómenos ambos que están haciendo disminuir sensiblemente la capacidad de prestación de cuidados informales, haciendo que el modelo de apoyo informal, que ya ha empezado a hacer crisis, sea insostenible a medio plazo. El nuevo papel social de las mujeres ha venido acompañado de profundas transformaciones en la estructura familiar, entre las que destacan una importante reducción de tamaño de los hogares y la diversificación de las formas de convivencia. Aunque el modelo predominante sigue siendo la familia conyugal nuclear, ésta se encuentra en proceso de disminución en la medida en que se ha producido un fuerte aumento de otros tipos de modelos de convivencia.

\section{ACTIVIDADES DE LA VIDA DIARIA (AVD)}

El primer problema que se plantea a la hora de estudiar la población dependiente es identificar cuáles son esos actos corrientes de la vida diaria que deben ser tenidos en cuenta a la hora de evaluar si una persona se encuentra 0 no en situación de dependencia, por necesitar una ayuda o asistencia importante para realizarlos. Para ello, puede utilizarse un concepto utilizado desde hace tiempo por disciplinas como la rehabilitación o la geriatría, el de "actividades de la vida diaria” (AVD), que son aquellas actividades que una persona ha de realizar diariamente para poder vivir de forma autónoma, integrada en su entorno habitual y cumpliendo su rol social. Algunos autores (Querejeta et al 2004) han definido y clasificado estas actividades en, básicas de la vida diaria (ABVD) e instrumentales de la vida diaria (AIVD).

- Actividades básicas de la vida diaria (ABVD): Son las actividades imprescindibles para poder subsistir de forma independiente. Entre ellas se incluyen las actividades de auto cuidado, asearse, vestirse y desnudarse, poder ir solo al servicio, poder quedarse solo durante la noche, comer y de funcionamiento básico físico y mental, desplazarse dentro del hogar $\mathrm{y}$ reconocer personas y objetos, orientarse, entender y ejecutar instrucciones y/o tareas sencillas.

- Actividades instrumentales de la vida diaria (AIVD): son actividades más complejas que las ABVD, y su realización requiere de un mayor nivel de autonomía personal. Se asocian a tareas que implican la capacidad de tomar decisiones. En esta categoria se incluyen tareas domésticas, de movilidad, de administración del hogar y de la propiedad, como poder utilizar el teléfono, acordarse de tomar la medicación, cortarse las uñas de los pies, subir una serie de escalones, coger un autobús, un metro o un taxi, preparar la propia comida, comprar lo que se necesita para vivir, realizar actividades domésticas básicas (fregar los platos, hacer la cama, etc.), poder pasear, ir 
al médico, hacer papeleos y administrar el propio dinero, entre otras.

La EDDES 99 fue y es, sin duda, la fuente de información más adecuada sobre la que fundamentar el estudio de la población dependiente en España. La EDDES 99 adoptó como marco de referencia conceptual la Clasificación Internacional de Deficiencias, Discapacidades y Minusvalias (CIDDM) propuesta en 1980 por la Organización Mundial de la Salud (OMS).

En la EDDES se define la discapacidad como"una limitación de la capacidad humana que hace imposible 0 dificulta gravemente la actividad normal de la persona", se estableció un listado de actividades básicas que permitiera preguntar a los encuestados de 6 y más años cuál o cuáles de ellas era incapaz de o tenía dificultades para realizar. Esas actividades básicas se agrupan, a su vez, en categorías que se corresponden con los grupos de discapacidades sobre los que aporta información la encuesta. En concreto, se han contemplado 36 actividades agrupadas en diez categorias, de acuerdo con el esquema siguiente:

\section{Ver}

1.1. Percibir cualquier imagen.

1.2. Realizar tareas visuales de conjunto.

1.3. Realizar tareas visuales de detalle.

1.4. Ver en condiciones de iluminación pobres, diferenciar colores, etc.

\section{Oir}

2.1. Recibir cualquier sonido.

2.2. Audición de sonidos fuertes.

2.3. Escuchar el habla.

\section{Comunicarse}

\subsection{Comunicarse a través del habla.}

3.2. Comunicarse a través de lenguajes alternativos.

3.3. Comunicarse a través de gestos no signados.

3.4. Comunicarse a través de escrituralectura convencional.

\section{Aprender, aplicar conocimientos y desarrollar tareas}

4.1. Reconocer personas y objetos y orientarse.

4.2. Recordar informaciones y episodios.

4.3. Entender y ejecutar órdenes $\mathrm{y} / 0$ tareas sencillas.

4.4. Entender y ejecutar órdenes y/o tareas complejas.

\section{Desplazarse}

5.1. Cambiar y mantener las posiciones del cuerpo.

5.2. Levantarse, acostarse.

5.3. Desplazarse dentro del hogar.

\section{Utilizar brazos y manos}

6.1. Trasladar objetos no muy pesados.

6.2. Utilizar utensilios y herramientas.

6.3. Manipular objetos pequeños con manos y dedos.

\section{Desplazarse fuera del hogar}

7.1. Deambular sin medio de transporte.

7.2. Desplazarse en transporte público.

7.3. Conducir vehículo propio (entre $18 \mathrm{y}$ 75 años).

\section{Cuidar de sí mismo}

8.1. Asearse solo: lavarse y cuidar su aspecto.

8.2. Controlar las necesidades y utilizar solo el servicio.

8.3. Vestirse-desvestirse y arreglarse.

8.4. Comer y beber.

9. Realizar las tareas del hogar (mayores de 10 años) 
9.1. Hacer compras y controlar los suministros y servicios.

9.2. Cuidarse de las comidas.

9.3. Limpieza y cuidado de la ropa.

9.4. Limpieza y mantenimiento de la casa.

9.5. Cuidarse del bienestar del resto de la familia.

\section{Relacionarse con otras personas}

10.1. Mantener relaciones de cariño con familiares.

10.2. Hacer nuevos amigos y mantener la amistad.

10.3. Relacionarse con compañeros, jefes, etc..

Obviamente, la elección de estas 36 actividades condiciona los resultados de la encuesta, pues si se hubieran elegido otras o si la lista hubiera sido más larga o más corta, las cifras globales de personas con discapacidad obtenidas a partir de la encuesta también habrían sido diferentes.

Las deficiencias estudiadas en la encuesta también se clasifican en tipos y categorías, de acuerdo con el siguiente esquema:

\section{DEFICIENCIAS MENTALES, VISUALES, AUDITIVAS, DEL LENGUAJE OSTEOARTICULARES, DEL SISTEMA NERVIOSO, VISCERALES Y OTRAS}

El Instituto Nacional de Estadística ha seleccionado, de entre el listado de 36 actividades básicas a través de las cuales se operativiza el concepto de discapacidad, 13 actividades que, por su relación con el autocuidado, la movilidad básica y las tareas domésticas, encajan dentro del concepto de "actividades de la vida diaria”.

Estas actividades son las siguientes:
- Realizar cambios de las diversas posiciones del cuerpo y mantenerlas.

- Levantarse, acostarse, permanecer de pie 0 sentado.

- Desplazarse dentro del hogar.

- Deambular sin medio de transporte.

- Asearse solo: lavarse y cuidarse de su aspecto.

- Controlar las necesidades e ir solo al servicio.

- Vestirse, desvestirse y arreglarse.

- Comer y beber.

- Cuidarse de las compras y del control de los suministros y servicios.

- Cuidarse de las comidas.

- Cuidarse de la limpieza y planchado de la ropa.

- Cuidarse de la limpieza y mantenimiento de la casa.

- Cuidarse del bienestar de los demás miembros de la familia.

Para cada una de las actividades afectadas se registra el nivel de dificultad que la persona encuestada tiene para realizarlas, lo que permite estimar la severidad de las discapacidades, en la escala siguiente:

- Discapacidad inexistente (sin dificultad alguna para realizar la actividad).

- Discapacidad moderada (con dificultad moderada para realizar la actividad).

- Discapacidad severa (con dificultad grave para realizar la actividad). 
- Discapacidad total (no puede realizar la actividad).

Basándose en estas 13 actividades y con referencia a la población mayor de 6 años, el INE estima el número de personas con alguna discapacidad para las actividades de la vida diaria es de 2.285.340. El número de personas con discapacidad severa o total para alguna de estas 13 actividades de la vida diaria se cifra en 1.547.195. Dentro de este listado de 13 actividades de la vida diaria utilizado por el INE no se incluyen algunas actividades relacionadas con el funcionamiento mental básico, como reconocer personas y objetos y orientarse o entender y ejecutar instrucciones $\mathrm{y} / 0$ tareas sencillas, que también son esenciales para un desenvolvimiento cotidiano mínimamente autónomo y como resultado en el estudio realizado se subestima el número de personas dependientes debido a trastornos mentales y demencias.
La esperanza de vida al nacer de los españoles, calculada de acuerdo con los datos proporcionados por la encuesta referida anteriormente, era en 1999 de 75,3 años para los varones y de 82,3 años para las mujeres. La esperanza de vida libre de enfermedades crónicas es, al nacer, de 40,9 años para los varones y de 37,9 para las mujeres. La esperanza de vida sin discapacidad puede cifrarse, desde el nacimiento, en 68,5 años para los varones y 72,12 años para las mujeres. Si comparamos estos índices con la esperanza de vida al nacer, podemos ver que los varones pasarán, como media, 6,8 años de su vida en situación de discapacidad, mientras que las mujeres vivirán, como media, 10,2 años con discapacidad. Si expresamos los años por vivir con discapacidad como porcentaje de la esperanza de vida total, vemos que estos suponen el 9 por cien de los años de vida en los varones, y el 12,4 por cien en las mujeres. La esperanza de vida sin discapacidades severas se estima, al nacimiento, en 71,1 años para los varones y en 75,4 años para las mujeres.

Cuadro 1. Personas con alguna discapacidad para las AVD por máximo grado de severidad y grupo de edad. España. 1999

\begin{tabular}{|l|r|r|r|r|}
\hline & 6 a 64 años & 65 a 79 años & 80 y más años & $\begin{array}{c}\text { Total mayores } \\
\text { de } 6 \text { años }\end{array}$ \\
\hline Discapacidad moderada & 287.610 & 279.230 & 126.977 & 693.817 \\
Discapacidad total & 258.241 & 307.792 & 165.672 & 731.705 \\
No consta & 261.547 & 257.455 & 296.489 & 815.491 \\
\hline Total & 13.127 & 17.942 & 13.257 & 44.326 \\
\hline
\end{tabular}

Fuente: Instituto Nacional de Estadística, Encuesta sobre Discapacidades, Deficiencias y Estado de Salud 1999, Resultados detallados. Madrid, 2002. 


\section{Cuadro 2. Personas con alguna discapacidad severa o total para las AVD} por tipo de discapacidad y grupo de edad. España. 1999

\begin{tabular}{|c|c|c|c|c|}
\hline & 6 a 64 años & 65 a 79 años & $\begin{array}{l}80 \text { y más } \\
\text { años }\end{array}$ & $\begin{array}{c}\text { Total } \\
\text { mayores de } 6 \\
\text { años }\end{array}$ \\
\hline $\begin{array}{l}\text { Cambiar y mantener las posiciones } \\
\text { del cuerpo }\end{array}$ & 150.083 & 183.163 & 157.194 & 490.440 \\
\hline Levantarse, acostarse, & 210.557 & 273.534 & 228.315 & 712.406 \\
\hline Desplazarse dentro del hogar & 143.445 & 220.507 & 231.573 & 595.525 \\
\hline Deambular sin medio de transporte & 282.015 & 414.863 & 385.586 & 1.082 .464 \\
\hline $\begin{array}{l}\text { Asearse solo: lavarse y cuidar su } \\
\text { aspecto }\end{array}$ & 149.542 & 182.279 & 260.695 & 592.516 \\
\hline $\begin{array}{l}\text { Controlar las necesidades y utilizar } \\
\text { solo el servicio }\end{array}$ & 72.489 & 84.619 & 150.020 & 307.128 \\
\hline Vestirse, desvestirse $y$ arreglarse & 139.017 & 158.578 & 217.535 & 515.130 \\
\hline Comer y beber & 54.415 & 52.599 & 91.639 & 198.653 \\
\hline $\begin{array}{l}\text { Compras y control de los } \\
\text { suministros y servicios }\end{array}$ & 304.106 & 354.128 & 374.629 & 1.032 .863 \\
\hline Cuidarse de las comidas & 192.758 & 215.501 & 305.848 & 714.107 \\
\hline Limpieza y cuidado de la ropa & 232.086 & 280.575 & 339.554 & 852.215 \\
\hline $\begin{array}{l}\text { Limpieza y mantenimiento de la } \\
\text { casa }\end{array}$ & 279.068 & 354.102 & 367.442 & 1.000 .612 \\
\hline $\begin{array}{l}\text { Cuidarse del bienestar del resto de } \\
\text { la familia }\end{array}$ & 207.149 & 214.409 & 288.016 & 709.574 \\
\hline Total & 519.787 & 565.247 & 462.161 & 1.547 .195 \\
\hline
\end{tabular}

Fuente: Instituto Nacional de Estadística, Encuesta sobre Discapacidades, Deficiencias y Estado de Salud 1999, Resultados detallados. Madrid, 2002.

La esperanza de vida sin discapacidad para las actividades de la vida diaria es, al nacer, de 72,8 años para los varones y de 75,4 años para las mujeres. Comparando estas cifras con la esperanza de vida total, el resultado es que, por término medio, los varones van a vivir 2,6 años de su vida en situación de dependencia, y las mujeres 5,2 años. A los 65 años, las esperanzas de vida sin discapacidad para las actividades de la vida diaria son de 13,9 años para los varones y de 15,6 años para las mujeres. Los años que, por término medio, vivirán en situación de dependencia las personas que han llegado con vida a los 65 años son, respectivamente, 2,2 y 4,7 , según se trate de varones o de mujeres. En el Libro Blanco publicado por IMSERSO se incluyen gráficos que muestran las curvas teóricas de supervivientes en diversas situaciones de salud y discapacidad.
La zona más oscura, comprendida entre la curva de supervivientes totales y la curva de supervivientes sin discapacidad para las actividades de la vida diaria representa gráficamente a las personas dependientes. Puede apreciarse con toda claridad como se incremen$\tan$ las situaciones de dependencia con la edad. La Encuesta sobre Deficiencias, Discapacidades y Estados de Salud de 1999 (EDDES 99) ha recogido información sobre las personas con discapacidad que reciben ayuda técnica (cuadro 5) y de asistencia personal (cuadro 6) por sistema proveedor de ayudas, grandes grupos de edad y sexo. España. 1999. Al igual que ocurre con muchas otras variables consideradas en la encuesta, de los datos publicados hacemos referencia a las ayudas personales especi-ficadas por el cuidador principal (cuadro 7). 
Cuadro 3. Esperanza de vida ajustada según estado de salud y discapacidad al nacer, a los 45 y a los 65 años. España. 1999

\begin{tabular}{|c|c|c|c|}
\hline & Al nacer & A los 45 años & A 10565 años \\
\hline \multicolumn{4}{|l|}{ Varones } \\
\hline Esperanza de vida & 75,29 & 32,70 & 16,17 \\
\hline EV libre de enfermedades crónicas & 40,85 & 10,17 & 3,31 \\
\hline EV en buena salud & 59,52 & 19,61 & 7,78 \\
\hline EV sin discapacidad & 68,52 & 26,90 & 11,39 \\
\hline EV sin discapacidad severa & 71,07 & 29,04 & 13,09 \\
\hline EV sin discapacidad para las AVD & 72,68 & 30,28 & 13,94 \\
\hline \multicolumn{4}{|l|}{ Mujeres } \\
\hline Esperanza de vida & 82,31 & 38,62 & 20,25 \\
\hline EV libre de enfermedades crónicas & 37,89 & 8,14 & 2,77 \\
\hline EV en buena salud & 58,17 & 18,25 & 7,52 \\
\hline EV sin discapacidad & 72,12 & 29,25 & 12,39 \\
\hline EV sin discapacidad severa & 75,44 & 32,18 & 14,66 \\
\hline EV sin discapacidad para las AVD & 77,07 & 33,53 & 15,56 \\
\hline
\end{tabular}

Fuente: INE. Encuesta sobre Discadacidades، Deficiencias v Estado de Salud. 1999.

Cuadro 4. Años por vivir en diferentes situaciones de salud y discapacidad al nacer, a los 45 y a los 65 años (en número de años y en porcentajes sobre la esperanza de vida a cada edad). España. 1999

\begin{tabular}{|c|c|c|c|c|c|c|}
\hline & $\begin{array}{r}A / n \\
\text { Años por } \\
\text { vivir }\end{array}$ & \begin{tabular}{|l} 
pacer \\
Poobrentaje \\
sobre EV
\end{tabular} & \begin{tabular}{|c|}
$A$ los \\
Años por \\
vivir
\end{tabular} & \begin{tabular}{|c|}
45 años \\
$\mid \begin{array}{c}\text { Porcentaje } \\
\text { sobre EV }\end{array}$
\end{tabular} & \begin{tabular}{|c|}
$A$ los \\
Años por \\
vivir
\end{tabular} & \begin{tabular}{|l|}
65 años \\
$\mid \begin{array}{c}\text { Porcentaje } \\
\text { sobre EV }\end{array}$
\end{tabular} \\
\hline \multicolumn{7}{|l|}{ Varones } \\
\hline Total años por vivir (EV) & 75,29 & 100,0 & 32,70 & 100,0 & 16,17 & 100,0 \\
\hline Con enfermedades crónicas & 34,44 & 45,7 & 22,53 & 68,9 & 12,86 & 79,5 \\
\hline Sin buena salud & 15,77 & 20,9 & 13,09 & 40,0 & 8,39 & 51,9 \\
\hline Con discapacidad & 6,77 & 9,0 & 5,80 & 17,8 & 4,78 & 29,6 \\
\hline Con discapacidad severa & 4,22 & 5,6 & 3,66 & 11,2 & 3,08 & 19,1 \\
\hline Con discapacidad para las AVD & 2,61 & 3,5 & 2,43 & 7,4 & 2,23 & 13,8 \\
\hline \multicolumn{7}{|l|}{ Mujeres } \\
\hline Total años por vivir (EV) & 82,31 & 100,0 & 38,62 & 100,0 & 20,25 & 100,0 \\
\hline Con enfermedades crónicas & 44,42 & 54,0 & 30,48 & 78,9 & 17,48 & 86,3 \\
\hline Sin buena salud & 24,14 & 29,3 & 20,37 & 52,7 & 12,73 & 62,9 \\
\hline Con discapacidad & 10,19 & 12,4 & 9,37 & 24,3 & 7,86 & 38,8 \\
\hline Con discapacidad severa & 6,87 & 8,3 & 6,44 & 16,7 & 5,59 & 27,6 \\
\hline Con discapacidad para las AVD & 5,24 & 6,4 & 5,08 & 13,2 & 4,69 & 23,2 \\
\hline
\end{tabular}

Fuente: INE, Encuesta sobre Discapacidades, Deficiencias y Estado de Salud, 1999. 
Cuadro 5 y 6. Personas con discapacidad que reciben ayudas de asistencia personal por sistema proveedor de ayudas, grandes grupos de edad y sexo (datos referidos a personas de 60 mas años). España. 1999.

\begin{tabular}{|l|r|r|r|r|}
\hline & 6 a 64 & 65 a 79 & \multicolumn{3}{|c|}{80 y más } & Total \\
\hline Ambos sexos & 112.230 & 133.035 & 86.778 & 332.043 \\
\hline Público & 141.402 & 233.609 & 175.749 & 550.760 \\
Privado con fines de lucro & 33.445 & 51.600 & 43.495 & 128.540 \\
Otro sistema privado & 15.092 & 15.721 & 12.093 & 42.906 \\
No consta & $\mathbf{2 8 3 . 5 6 9}$ & $\mathbf{4 1 0 . 8 0 5}$ & $\mathbf{2 9 6 . 8 8 6}$ & $\mathbf{9 9 1 . 2 6 0}$ \\
Total & \multicolumn{4}{|c|}{} \\
\hline Varones & 60.096 & 50.128 & 25.740 & 135.964 \\
\hline Público & 73.899 & 93.930 & 63.606 & 231.435 \\
Privado con fines de lucro & 19.149 & 20.620 & 13.349 & 53.118 \\
Otro sistema privado & 7.556 & 6.584 & 2.726 & 16.866 \\
No consta & $\mathbf{1 5 0 . 0 3 0}$ & $\mathbf{1 6 2 . 7 8 7}$ & $\mathbf{9 9 . 2 4 6}$ & $\mathbf{4 1 2 . 0 6 3}$ \\
Total & 52.135 & 82.907 & 61.038 & 196.080 \\
\hline Mujeres & 67.503 & 139.679 & 112.143 & 319.325 \\
\hline Público & 14.297 & 30.980 & 30.147 & 75.424 \\
Privado con fines de lucro & 7.536 & 9.136 & 9.367 & 26.039 \\
Otro sistema privado & $\mathbf{1 3 3 . 5 3 9}$ & $\mathbf{2 4 8 . 0 1 8}$ & $\mathbf{1 9 7 . 6 4 0}$ & $\mathbf{5 7 9 . 1 9 7}$ \\
No consta & \multicolumn{3}{|c}{} \\
Total &
\end{tabular}

Fuente: Instituto Nacional de Estadística, Encuesta sobre Discapacidades, Deficiencias y Estado de Salud 1999, Resultados detallados. Madrid, 2002.

\begin{tabular}{|c|c|c|c|c|}
\hline & $6 a 64$ & $65 \therefore 79$ & 80 y más & Total \\
\hline \multicolumn{5}{|l|}{ Ambos sexos } \\
\hline Público & 39.641 & 39.015 & 44.489 & 123.145 \\
\hline Familia & 434.718 & 467.453 & 408.421 & 1.310 .592 \\
\hline Otro sistema privado & 48.364 & 89.060 & 84.060 & 221.484 \\
\hline No consta & 90.663 & 73.909 & 45.424 & 209.996 \\
\hline Total & 556.954 & 605.064 & 508.432 & 1.670 .450 \\
\hline \multicolumn{5}{|l|}{ Varones } \\
\hline Público & 22.159 & 10.806 & 9.462 & 42.427 \\
\hline Familia & 197.889 & 148.968 & 109.244 & 456.101 \\
\hline Otro sistema privado & 13.221 & 15.888 & 16.625 & 45.734 \\
\hline No consta & 48.732 & 28.886 & 14.448 & 92.066 \\
\hline Total & 258.210 & 189.508 & 132.961 & 580.679 \\
\hline \multicolumn{5}{|l|}{ Mujeres } \\
\hline Público & 17.482 & 28.210 & 35.026 & 80.718 \\
\hline Familia & 236.829 & 318.485 & 299.177 & 854.491 \\
\hline Otro sistema privado & 35.143 & 73.173 & 67.435 & 175.751 \\
\hline No consta & 41.931 & 45.023 & 30.976 & 117.930 \\
\hline Total & 298.744 & 415.556 & 375.471 & 1.089 .771 \\
\hline
\end{tabular}

Fuente: Instituto Nacional de Estadística, Encuesta sobre Discapacidades, Deficiencias y Estado de Salud 1999, Resultados detallados. Madrid, 2002. 
Cuadro 7. Personas con discapacidad que reciben ayudas de asistencia personal en relación con el cuidador principal. España. 1999

\begin{tabular}{|c|c|c|c|c|}
\hline & $6 a 64$ & $65+79$ & 80 y mís & Tatal \\
\hline \multicolumn{5}{|l|}{ Ambos sexos } \\
\hline $\begin{array}{l}\text { Cónyuge } \\
\text { Hija }\end{array}$ & $\begin{array}{r}146.783 \\
65.378\end{array}$ & $\begin{array}{l}193.791 \\
151.500\end{array}$ & $\begin{array}{r}50.649 \\
200.285\end{array}$ & $\begin{array}{l}391.223 \\
417.163\end{array}$ \\
\hline $\begin{array}{l}\text { Hijo } \\
\text { Hermana }\end{array}$ & $\begin{array}{l}17.019 \\
18.608\end{array}$ & $\begin{array}{l}30.796 \\
16.926\end{array}$ & $\begin{array}{r}31.952 \\
8.944\end{array}$ & $\begin{array}{l}79.767 \\
44.478\end{array}$ \\
\hline Hermano & 5.681 & 2.128 & 1.191 & 9.000 \\
\hline Madre & 140.094 & 322 & 0 & 140.416 \\
\hline Padre & 12.481 & 0 & 0 & 12.481 \\
\hline Otro periente & 27.415 & 63.330 & 94.119 & 184.864 \\
\hline Empleado & 17.957 & 46.548 & 48.188 & 112.693 \\
\hline Amigos y vecinos & 4.939 & 12.566 & 9.140 & 26.645 \\
\hline $\begin{array}{l}\text { Servicios sociales } \\
\text { Otra relación }\end{array}$ & $\begin{array}{r}16.286 \\
1.507\end{array}$ & $\begin{array}{r}16.384 \\
4.423\end{array}$ & $\begin{array}{r}18.217 \\
8.933\end{array}$ & $\begin{array}{l}50.887 \\
14.863\end{array}$ \\
\hline $\begin{array}{l}\text { No consta } \\
\text { Total }\end{array}$ & $\begin{array}{r}82.807 \\
556.954\end{array}$ & $\begin{array}{r}66.349 \\
605.064\end{array}$ & $\begin{array}{r}36.816 \\
508.432\end{array}$ & $\begin{array}{r}185.972 \\
\mathbf{1 . 6 7 0 . 4 5 0}\end{array}$ \\
\hline \multicolumn{5}{|l|}{ Varones } \\
\hline Cómyuge & 79.155 & 102.938 & 35.688 & 217.781 \\
\hline Hija & 5.688 & 23.016 & 42.576 & 71.280 \\
\hline Hijo & 2.116 & 7.078 & 6.725 & 15.919 \\
\hline Hemana & 9.261 & 4.832 & 1.533 & 15.626 \\
\hline Hemano & 4.078 & 611 & 0 & 4.689 \\
\hline Madre & 81.292 & 322 & 0 & 81.614 \\
\hline Padre & 9.353 & 0 & 0 & 9.353 \\
\hline Otro periente & 9.574 & 12.152 & 17.377 & 39.103 \\
\hline Empleado & 1.856 & 7.074 & 10.381 & 19.311 \\
\hline Amigos y vecinos & 1.349 & 2.546 & 1.032 & 4.927 \\
\hline Servicios sociales & 8.896 & 2.728 & 3.850 & 15.474 \\
\hline Otra relación & 976 & 1.116 & 1.505 & 3.597 \\
\hline No consta & 44.615 & 25.096 & 12.295 & 82.006 \\
\hline Total & 258.210 & 189.508 & 132.961 & 580.679 \\
\hline \multicolumn{5}{|l|}{ Mujeres } \\
\hline Cónyuge & 67.628 & 90.853 & 14.961 & 173.442 \\
\hline Hija & 59.690 & 128.484 & 157.708 & 345.882 \\
\hline Hijo & 14.902 & 23.719 & 25.227 & 63.848 \\
\hline Hermans & 9.347 & 12.094 & 7.411 & 28.852 \\
\hline Hemano & 1.603 & 1.517 & 1.191 & 4.311 \\
\hline Madre & 58.803 & 0 & 0 & 58.003 \\
\hline Padre & 3.128 & 0 & 0 & 3.128 \\
\hline Otro pariente & 17.840 & 51.178 & 76.742 & 145.760 \\
\hline Empleado & 16.101 & 39.474 & 37.806 & 93.381 \\
\hline Amigos y vecinos & 3.590 & 10.019 & 8.108 & 21.717 \\
\hline Servicios sociales & 7.389 & 13.656 & 14.367 & 35.412 \\
\hline Otra relacóón & 531 & 3.308 & 7.428 & 11.267 \\
\hline No consta & 38.192 & 41.253 & 24.521 & 103.966 \\
\hline Total & 298.744 & 415.556 & 375.471 & 1.089 .771 \\
\hline
\end{tabular}

Fuente: Instituto Nacional de Estadística, Encuesta sobre Discapacidades, Deficiencias y Estado de Salud 1999. Resultados detallados. Madrid. 2002. 


\section{SISTEMA NACIONAL DE DEPENDENCIA Y DEFINICIÓN DEL "UMBRAL DE ENTRADA"}

Cualquier estimación del esfuerzo que habrá de realizar el futuro Sistema Nacional de Dependencia ha de partir de una definición del "umbral de entrada" o "pérfil de inclusión" en el sistema y de una fórmula de graduación de los niveles de dependencia que habrán de servir de referencia para la determinación de la cuantía de las prestaciones o para fijar los objetivos en cuanto a la intensidad y frecuencia de los servicios de atención.

Como nos muestra la experiencia de los países de nuestro entorno, pueden adoptarse diferentes opciones a la hora de fijar el umbral de entrada y de establecer los distintos niveles de protección.

Así, por ejemplo, el modelo alemán establece el umbral de entrada en función de la cantidad de ayuda que las personas dependientes precisan, considerando que se da una situación de dependencia cuando la persona necesita como mínimo 90 minutos al día de atención, la mitad de este tiempo para cuidados personales, y establece, a partir de ahí, tres grados de dependencia.

El modelo francés, por su parte, establece el umbral de entrada en función del tipo de ayuda necesaria, considerando que existe dependencia cuando una persona necesita ayuda para asearse y vestirse, para realizar actividades personales o para alimentarse, y también cuando necesita ayuda para levantarse de la cama o de una silla, aunque pueda desplazarse sola en el interior del domicilio. A diferencia del sistema alemán, el subsidio personalizado de autonomía francés sólo cubre a las personas mayores de 60 años.
El criterio de umbral de entrada en el Sistema Nacional de Dependencia Español, así como los grados o niveles de dependencia que gozarán de protección habrán de fijarse, al igual que se ha hecho en los países de nuestro entorno, en función de la cantidad y del tipo de ayuda que la persona necesite.

Partimos también de la hipótesis de que se establecerán tres grados de dependencia, que provisionalmente podríamos denominar dependencia moderada, dependencia severa y gran dependencia. Ello permitirá graduar la dependencia, discriminando las situaciones de menor a mayor intensidad y facilitando a la vez la valoración y la gestión de la prestación.

A continuación se detallan los criterios que señala el libro blanco de la Dependencia.

- Grado 3 (gran dependencia): Cuando la persona necesita ayuda para realizar varias actividades básicas de la vida diaria varias veces al día $y$, por su pérdida total de autonomía mental o física, necesita la presencia indispensable y continua de otra persona.

- Grado 2 (dependencia severa): Cuando la persona necesita ayuda para realizar varias actividades básicas de la vida diaria dos o tres veces al día, pero no requiere la presencia permanente de un cuidador.

- Grado 1 (dependencia moderada): Cuando la persona necesita ayuda para realizar una 0 varias actividades básicas de la vida diaria, al menos una vez al día.

Las personas que, sin tener una discapacidad en grado severo o total para las actividades básicas de la vida diaria, 
tienen necesidad de ayuda para realizar tareas domésticas y/o discapacidad moderada en áreas de autocuidado, movilidad, alimentación y funcionamiento mental básico, se han distribuido en dos grupos, de las siguientes características:

- Grupo A: Personas con discapacidad moderada para alguna actividad básica de la vida diaria que no necesitan ayuda diariamente.

- Grupo B: Personas con discapacidad para alguna actividad instrumental de la vida diaria (movilidad fuera del hogar y tareas domésticas) que no tienen discapacidad para ninguna actividad básica.

\section{MODELO DE ESTIMACIÓN}

Para la estimación de la población encuadrada en cada grado de dependencia (Libro Blanco, Sistema Nacional de Dependencia) se ha partido de la población que tienen una discapacidad severa 0 total para alguna de las nueve actividades básicas de la vida diaria consideradas.

Una vez seleccionada la población, se ha elaborado un indicador sintético de la necesidad de ayuda que tiene en cuenta el número de actividades básicas de la vida diaria afectadas y el grado de discapacidad para cada una de ellas, incluyendo no sólo las discapacidades severas o totales, sino también las moderadas:

- Por cada actividad respecto de la cual la persona presenta una discapacidad moderada se ha asignado un punto.

- Por cada actividad respecto de la cual la persona presenta una disca- pacidad severa, se han asignado dos puntos.

- Por cada actividad respecto de la cual la persona presenta una discapacidad total, se han asignado tres puntos.

A partir de la puntuación, las personas dependientes se han encuadrado en cado uno de los grados de dependencia de acuerdo a los siguientes criterios:

- Grado 3 (gran dependencia): más de 15 puntos.

- Grado 2 (dependencia severa): entre 7 y 15 puntos.

- Grado 1 (dependencia moderada): menos de 7 puntos.

Los criterios para el encuadramiento de las personas que, sin tener una discapacidad en grado severo o total para las actividades básicas de la vida diaria, tienen necesidad de ayuda para realizar tareas domésticas y/o discapacidad moderada en áreas de autocuidado, movilidad, alimentación y funcionamiento mental básico, han sido los siguientes:

- Grupo A: Personas que tienen al menos una discapacidad moderada para alguna actividad básica de la vida diaria, siempre que no presentan discapacidad severa o total para ninguna otra actividad básica.

- Grupo B: Personas que tienen alguna discapacidad de cualquier grado para alguna actividad instrumental de la vida diaria, siempre que no tengan discapacidad para ninguna actividad básica de la vida diaria.

Los cinco grupos así definidos son mutuamente excluyentes, de manera 
que una persona no puede estar clasificada simultáneamente en dos o más de ellos.

\section{LOS OBJETIVOS DE LA ATENCIÓN}

El IMSERSO define, como objetivo general de la atención, el "facilitar y potenciar el mantenimiento de la autonomía de la persona mayor". Si el deterioro de sus capacidades es tan grave que se produce, junto a la pérdida de independencia, la pérdida de la autonomía, el objetivo será atender a la persona mayor con las máximas garantias de respeto y dignidad debidos a cada individuo de la especie humana. Entre los objetivos a conseguir con los profesionales que atienden a los dependientes se encuentran.

Fomentar el desarrollo de investigaciones científicas relacionadas con estos programas, así como el controlar y seguir terapéuticamente las enfermedades y trastornos detectados mejorando y manteniendo, hasta donde sea posible, la capacidad funcional y mental, mediante técnicas rehabilitadoras, previniendo además el incremento de la dependencia mediante terapias y programas.

\section{LA ATENCIÓN SANITARIA}

La atención sanitaria a las personas dependientes es un factor clave por los siguientes motivos:

- Las enfermedades son, con gran diferencia, la causa principal de la dependencia.

- En muchas ocasiones esas enfermedades se han podido prevenir.

- La mayoría de las enfermedades que ya están causando dependencia requieren controles y tratamientos para evitar, entre otras cosas, que la dependencia progrese.

- Son muy frecuentes las descompensaciones de las patologías crónicas, al igual que las patologias múltiples, con su polifarmacia añadida, etc., lo que conlleva a una especial dificultad en el manejo asistencial (domicilio, residencia, hospital) y clínico (diferentes especialidades) de estos pacientes.

- Con frecuencia son los problemas sanitarios de las personas mayores dependientes los que desbordan las situaciones de asistencia. Si bien es necesario reforzar los dispositivos asistenciales para las personas gravemente dependientes, no se deben dejar en segundo plano los esfuerzos para evitar la dependencia o para la recuperación cuando la discapacidad es reversible, por lo que las intervenciones que tengan como objetivo la actuación preventiva, precoz y rehabilitadora deben tener un lugar preferente. La dependencia se asocia al envejecimiento, pero afecta también a personas que han sufrido accidentes, enfermedades de distinto tipo, malformaciones congénitas, etc. Todas ellas son susceptibles de recibir cuidados sanitarios y sociales hasta ahora no suficientemente desarrollados.

Las prestaciones sanitarias se han centrado, principalmente, en la atención con fines curativos. Sin embargo, el envejecimiento demográfico, la mayor prevalencia de las enfermedades crónicas como consecuencia de la mayor capacidad resolutiva del sistema, la mayor supervivencia de personas con discapacidad, la mayor esperanza de vida de las personas mayores y el des- 
arrollo social, determinan nuevas demandas en la cobertura, el funcionamiento y la utilización de los servicios sanitarios. El incremento de personas con dependencia que esto supone, llevará consigo la necesidad de desarrollar un sistema de Atención a la Dependencia que cubra los aspectos de cuidado social y los relacionados con la cobertura de las necesidades sanitarias que las personas con dependencia puedan presentar.

\section{BIBLIOGRAFÍA}

Assous, Laurence y Ralle, Pierre (2000c). Le prise en charge de la dependance des personnes agées: une mise en perspective internationale. Association Internationales de la Securité Sociale. (AISS).

Bebbington, A y M. Bone (1998), Healthy Life Expectancy and Long Term Care Discussion Paper 1426. PSSRU, University of K ent.

Belleti, F y H. K een (1998), Social protection for dependency in old age in Italy. Leuven: HIVA.

Breuil-Genier, Pascale (1999): "Aide aux personnes âgées dépendantes: une très grande hétérogénéité des pratiques» in Comparer les systèmes de protection sociale en Europe du Nord et en France, Rencontres de Copenhague, vol. 1, MIRE-DREES.

Challis, D. (1992), "The care of the elderly in Europe: social care", European Journal of Gerontology, 1.

Daatland, S.O. (1997a): Social protection for the elderly in Norway. Oslo,NOVA-skriftserie 4/97.

Depérée, Nicole (2000). Politiques europeennes de prise en charge de la dependance. Gerontologie et societé, no 93 , junio 2000.Madrid, Consejo Superior de Investigaciones Cientificas.

European Commission (2003,c) Feasibility Study. Comparable statistics in the area of care of dependent adults in the European Union.Luxemburg.

Evers, A. (1996), El nuevo seguro de asistencia a largo plazo en Alemania: características, consecuencias y perspectivas, Revista Española de Geriatria y Gerontología, no ${ }^{\circ} 6$.
Ferrera, M., HERMERIJCK, A.; RHODES, M. (2000) The future of SocialEurope: recasting work and welfare in the new economy. Report for the Portuguese Presidency of the European Union.

Hansen, E. B. (1998), Social protection for dependency in old age in Denmark. Leuven: HIVA.

IMSERSO. (1999) Vejez y Protección Social de la Depè ndencia en Europa.Observatorio de Personas Mayores. Ministerio de Trabajo y Asuntos Sociales.Madrid.

IMSERSO (1999): La Protección Social de la Dependencia. ColecciónServicios Sociales, no 42 . Ministerio de Trabajo y Asuntos Sociales, Madrid.

Jacobzone, S. Et al . (1998), Long Term Care Services to Older People, a perspective on future needs: the impact of an improving health of older persons. París: OCDE. Capítulo XI- Pág. 76

Jacobzone, Stephane (1999): "Le vieillissement et les soins pour les personnes âgées dépendantes: un aperçu des perspectives internationales" , OECD Labour market and social policy occasional papers, $n^{\circ} 38$.

Jenson, J. Y S. Jacobzone (2000), Care allowances for the frail elderly people and their impact on women care-givers. Paris: OCDE.

Mire (1997): Comparer les systèmes de protection sociale en Europe du S ud,Rencontres de Florence, $612 \mathrm{p}$.

Missoc (2001): La protection sociale dans les Etats membres de l'ue et del' Espace économique européen. Situation au janvier 2001. 
Montserrat, J. (2004) Los costes de la protección social de la dependencia para la población mayor: gasto razonable versus gasto actual. En D.Casado (dir.) Respuestas a la Dependencia. Madrid: E. CCS.

Nossosco (1998): Données statistiques, Nordic Social-Statistical Committee

Pacolet, J. Et al . (1998), Social Protection for Dependency in old age in the $15 \mathrm{EU}$ Member States and Norway. Bruselas: European Commission.

Rodríguez Cabrero, G (2004) Protección Social de la Dependencia en España. Madrid: Fundación Alternativas.

Rodríguez Cabrero, G. (2004) Referencias europeas de la protección social de la dependencia. En D. Casado (dir.) Respuestas a la Dependencia. Madrid: E. CCS

Rodríguez Castedo, A. (2002). Regulación de la atención a la dependencia en la Unión Europea. Modelos comparados. Universidad de Valencia. Royal Commission (1999), Long-term care, rights and responsabilities,Londres: HMSO.Capítulo XIPág. 77

Scharf, T. Y C. Wenger (eds.) (1995), International Perspectives on Community Care for Older People. Avebury: Aldershot.

Schneider, U. (1999). L"a ssurance sociale de soins longue durée en Allemagne:conception, application et evaluation. En revue internationale de sécurité sociale, vol.52. 2/99.

Schulte, B. (1996), Social protection for dependence in old age: the case of Germany, en Eisen, R. Y F.A. Sloan (ed.), Long-term care: economic issues and policy solutions. Boston/ Dordrecht/Londres: Kluwer.

Threfall, M. (2003) European social integration: harmonization, convergence and single social areas. Journal of European Social Policy, , vol.13, n.2.

Walker, A y L. Warren (1994), The case of frail older in Britain: current policies and future prospects, en OLSEN, K.K.: The graying of the World ,Nueva York y Winnipeng : Centre for Health Policy and Evaluation. 